\title{
Vertebral Augmentation for Neoplastic Lesions with Posterior Wall Erosion and Epidural Mass
}

\author{
A. Cianfoni, E. Raz, S. Mauri, S. Di Lascio, M. Reinert, G. Pesce, and G. Bonaldi
}

\begin{abstract}
BACKGROUND AND PURPOSE: The presence of a cortical erosion of the posterior wall or an epidural mass is commonly considered a contraindication to performing a vertebral augmentation, considering the perceived increased risk of an epidural cement leak. Our aim was to assess technical and clinical complications of vertebral augmentation procedures performed for pain palliation and/or stabilization of neoplastic lytic vertebral body lesions, with cortical erosion of the posterior wall, often associated with a soft-tissue epidural mass.
\end{abstract}

MATERIALS AND METHODS: In 48 patients, we performed retrospective vertebral augmentation assessment on 70 consecutive levels with cortical erosion of the posterior wall, as demonstrated by preprocedural CT/MR imaging. An epidural mass was present in 31/70 (44.3\%) levels. Cavity creation was performed with Coblation Wands before cement injection in 59/70 levels. Injection of high-viscosity polymethylmethacrylate was performed under real-time continuous fluoroscopic control. Postprocedural CT of the treated levels was performed in all cases. Clinical follow-up was performed at 1 and 4 weeks postprocedurally.

RESULTS: In 65/70 (92.8\%) levels, the vertebral augmentation resulted in satisfactory polymethylmethacrylate filling of the lytic cavity and adjacent trabecular spaces in the anterior half of the vertebral body. An epidural leak of polymethylmethacrylate occurred in 10/70 (14.2\%) levels, causing radicular pain in 3 patients, which spontaneously resolved within 1 week in 2 patients, while 1 patient with a T1-T2 foraminal leak developed severe weakness of the intrinsic hand muscles and a permanent motor deficit.

CONCLUSIONS: In our series of vertebral augmentation of neoplastic lytic vertebral lesions performed for palliation of pain and/or stabilization, we observed a polymethylmethacrylate epidural leak in only $14.2 \%$ of levels, despite the presence of cortical erosion of the posterior wall and an epidural mass, with an extremely low rate of clinical complications. Our data seem to justify use of vertebral augmentation in patients with intractable pain or those at risk for vertebral collapse.

ABBREVIATIONS: $\mathrm{CE}-\mathrm{PW}=$ cortical erosion of the posterior wall; $\mathrm{EM}=$ epidural mass; $\mathrm{PMMA}=$ polymethylmethacrylate; $\mathrm{VA}=$ vertebral augmentation

$S^{\mathrm{s}}$ pinal metastases, which are present in approximately $5 \%$ of patients with systemic cancer, ${ }^{1,2}$ are often associated with severe pain and reduced quality of life and can lead to potential catastrophic complications linked to spinal cord compression. ${ }^{3}$

Vertebroplasty, first described in $1987,{ }^{4}$ and, in more general terms, vertebral augmentation (VA) procedures are widely performed, especially for osteoporotic fractures ${ }^{5}$ but also as palliative treatment for painful or at-risk-of-collapse neoplastic lytic vertebral

Received May 6, 2014; accepted after revision June 26.

From the Departments of Neuroradiology (A.C., E.R.) and Neurosurgery (M.R.), Neurocenter of Southern Switzerland, Lugano, Switzerland; Department of Radiology (E.R.) New York University Langone Medical Center, New York, New York; Department of Neurology and Psychiatry (E.R.), Sapienza University of Rome, Rome, Italy; Oncology Institute of Italian Switzerland (S.M., S.D.L., G.P.), Bellinzona, Switzerland; and Department of Neuroradiology (G.B.), Papa Giovanni XXIII Hospital, Bergamo, Italy.

Please address correspondence to Eytan Raz, MD, Department of Radiology, NYU Langone Medical Center, 660 First Ave, 7th floor, 10016 New York, NY; e-mail: eytan.raz@gmail.com

http://dx.doi.org/10.3174/ajnr.A4096

body lesions. ${ }^{6}$ The intent of the procedure is to relieve pain and/or stabilize the vertebral body to arrest a developing fracture or prevent a new one. ${ }^{7-9}$ Multiple recent studies have shown a significant improvement in clinical outcomes (including pain) by using percutaneous vertebroplasty with or without kyphoplasty. ${ }^{10-12}$ The presence of a cortical erosion of the posterior wall (CE-PW) or an epidural mass (EM) is commonly considered a contraindication to performing VA, considering the perceived increased risk of an epidural polymethylmethacrylate (PMMA) cement leak. ${ }^{13}$ A PMMA epidural leak carries the risk of compression of the spinal cord and/or the nerve roots. The cement may also displace the tumor posteriorly, through a dehiscent posterior wall into the spinal canal; this event may be an even more dangerous complication, given the lack of control and visualization under fluoroscopy. ${ }^{13}$

The recently introduced controlled ablation (Coblation; ArthroCare, Austin, Texas) is a technique that allows the destruction of the tumor by placing a radiofrequency "wand" through the 

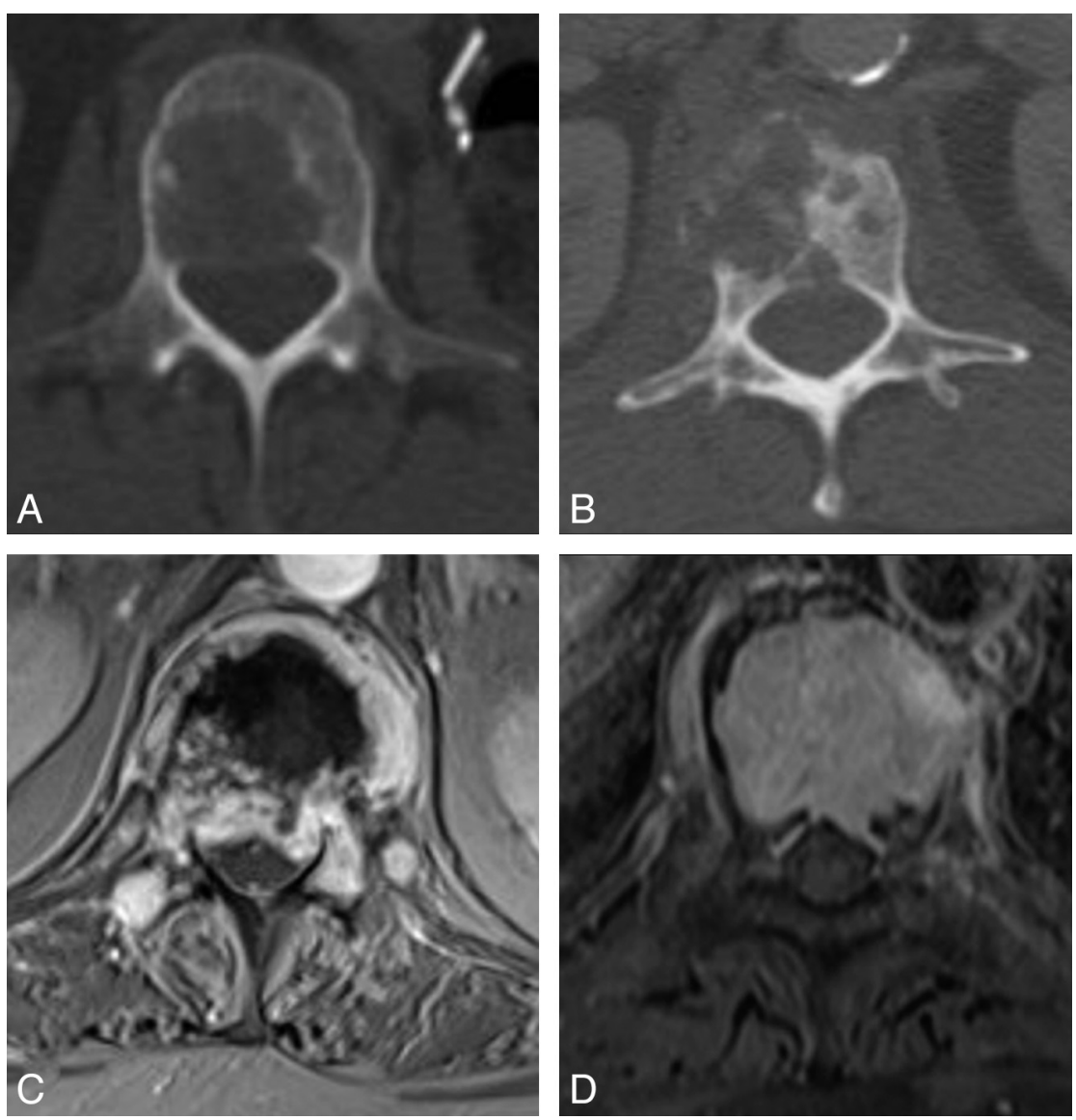

FIG 1. Preprocedural imaging. All patients had preprocedural CT to detect the presence of lytic cortical erosion of the posterior wall of the vertebral body, which was dichotomized into 2 main patterns: large defect $(A)$ and permeative erosive defect $(B)$. All patients had preprocedural MR imaging of the spine at the target levels to detect extraosseous neoplastic epidural soft tissue, dichotomized into large and convex $(C)$ and small and concave $(D)$.

vertebroplasty needle and uses the activation of a plasma field to vaporize the neoplastic soft tissue and create a cavity ${ }^{14}$; this cavity can then be filled more precisely and with low-pressure injection with PMMA, with a reduced risk of cement leak and posterior tumor displacement. ${ }^{15,16}$ The use of high-viscosity PMMA has a rationale in the treatment of such lesions, having been reported to help reduce the rate of extravertebral cement leak in benign and malignant fractures. ${ }^{17}$

The aim of this work was to assess technical and clinical complications of VA, performed for pain palliation and/or stabilization of neoplastic lytic vertebral body lesions, in a cohort of patients with CE-PW with or without associated soft-tissue EM.

\section{MATERIALS AND METHODS}

\section{Patients}

Patients were eligible for this retrospective, institutional review board-approved study if they were treated with VA between March 2011 and December 2012. At the time of treatment, patients were entered into a prospectively maintained data base. Additional data were collected by reviewing charts and reports and by analyzing all radiologic images available. For this analysis, we selected the subgroup of patients treated for a malignant vertebral lytic lesion with CE-PW. Indications for treatment were pain palliation and/or stabilization of neoplastic lytic vertebral body lesions. All patients underwent preprocedural CT and gadoliniumenhanced MR imaging of the spine at the target level.

Two neuroradiologists evaluated, in consensus, the preprocedural CT to determine the presence or absence of CE-PW and the preprocedural MR imaging to determine the presence of EM. The study population was, hence, a series of procedures on 70 consecutive vertebral levels in 48 patients. The mean age of the included patients was 64 years (range, 36-84 years; 20 men and 28 women). We recorded the following lesion characteristics: primary tumor, vertebral level, presence of an EM, dichotomic differentiation of the cortical erosion as "large" or "permeative," dichotomic differentiation of the EM as "large and convex" or "small and concave" (Fig 1), and the presence of compression of neural structures.

The VA procedures were variably performed before, during, or after radiation treatment and/or chemotherapy, following a multidisciplinary shared individualized clinical decision among a neuroradiologist, neuro-oncologist, radiation oncologist, and neurosurgeon.

\section{Percutaneous VA Procedure and Follow-Up}

All procedures were performed by the same operator, with variable participation of trainees under supervision. All patients were under monitor-assisted-care intravenous sedation and local anesthesia or under general anesthesia. The procedural technique was individualized and varied for different patients, at the operator's discretion, depending on the morphology, extent, consistency, and level of the vertebral lesions (Fig 2). The VA procedures were all performed under fluoroscopic guidance and control by using single-plane equipment. Combined additional CT guidance was used for some procedures to assist in accurate needle placement, mainly in the presence of extensive lytic changes involving the pedicles. In those cases, a $\mathrm{C}$-arm fluoroscope was positioned in a lateral view, just adjacent to the CT gantry, to monitor PMMA injection in real-time. Intraoperative myelography was used during treatment of some vertebrae in the lumbar or thoracolumbar junction areas in the presence of a large soft-tissue EM projecting into the spinal canal (Fig 3).

Beveled 15-, 10-, or 8-ga bone needles were inserted into the vertebral body under fluoroscopic guidance. A unilateral or bilateral transpedicular or parapedicular approach was used in the thoracolumbar spine. In the cervical spine, the anterolateral approach was used for $\mathrm{C} 4-\mathrm{C} 7$ levels, while a transoral approach was used for C2-C3 levels and a posterolateral CT-guided approach 

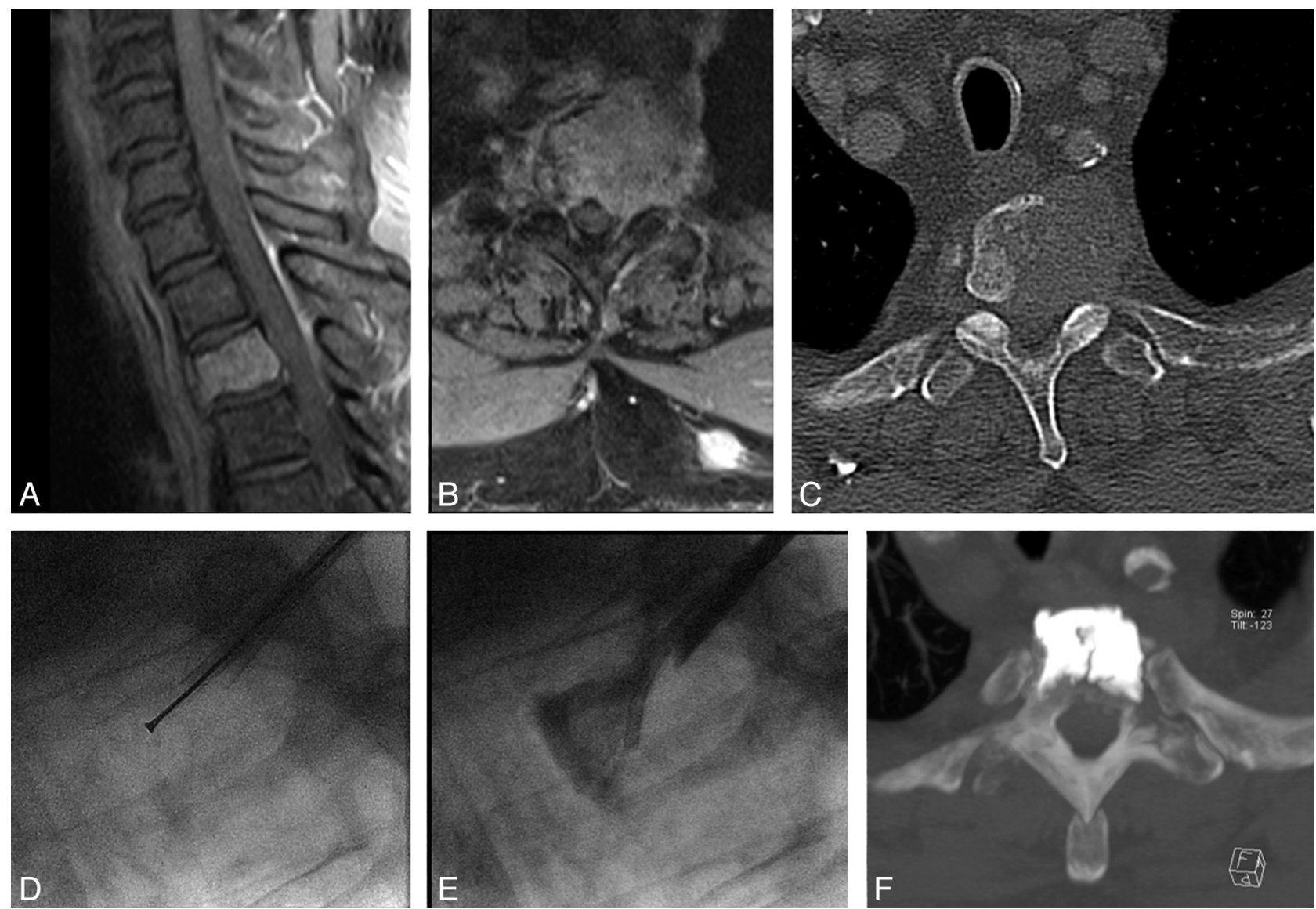

FIG 2. Cement augmentation of the weight-bearing portion of the vertebral body. Extensive lung cancer metastatic lytic destruction of the T2 vertebral body, with large cortical erosion of the posterior wall and a small concave epidural mass $(A-C)$ at risk of impending collapse. After cavity creation with Coblation $(D)$, we performed cement augmentation by injecting high-viscosity PMMA with a coaxial, curved, directional cannula $(E)$ to achieve cement distribution in the anterior two-thirds of the vertebral body, spanning superior-to-inferior disk endplates and preventing epidural leaks $(E-F)$. The cement filling was deemed excellent and appropriate for providing structural stability.

was used for the $\mathrm{C} 1$ level. ${ }^{18}$ Coaxially, several devices were variably inserted into the vertebral body. Plasma-field-activated radiofrequency Coblation Wands (ArthroCare), osteotomes, and curettes were used for cavity creation within the anterior twothirds of the vertebral body when deemed necessary and appropriate, especially for solid tumors, before cement injection, following the technique already described in the literature. ${ }^{19}$ When vertebral height restoration seemed desirable, in an attempt to reduce vertebral collapse, kyphoplasty balloons (Kyphon; Medtronic, Minneapolis, Minnesota) were used (Fig 3A-D). Finally, high-viscosity PMMA (Vertaplex HV; Stryker, Kalamazoo, Michigan) was injected in variable amounts under real-time continuous fluoroscopic control, until satisfactory filling of the lytic cavity and interdigitation in adjacent trabecular bone in the anterior two-thirds of the vertebral body were achieved. The aim was to obtain cement deposition in the portion of the vertebra at risk of collapse, namely the anterior two-thirds of the vertebral body, possibly spanning from superior-to-inferior endplates across the midline.

PMMA injection was halted if radiopaque cement approached the central canal or visibly tended to leak outside the vertebra, in the soft tissues, or in vascular structures. Control nonenhanced helical CT of the treated spinal segments was performed in every patient within 24 hours postprocedurally to assess PMMA leaks in the epidural space and foramina and to evaluate the PMMA filling of lytic cavities and adjacent trabecular spaces. CT datasets were reconstructed with a bone algorithm with 3-mm-thick multiplanar reformatted and 10-mm MIP images in the axial, sagittal, and coronal planes and were reviewed in consensus by 2 neuroradiologists. Clinical follow-up was performed at 1 week over the phone by a specialized nurse and at 4 weeks by a physician in consultation, to evaluate treatment-related clinical complications. The Visual Analog Scale pain score (range, 0-20) was measured before, 1 week after, and 1 month after treatment. Our clinical practice included plain film follow-up at 1 month postprocedure of the target spinal segment for those patients with nonsatisfactory (poor) cement filling, to rule out increased or newly developed vertebral collapse.

\section{Statistics}

The data are expressed as mean $\pm \mathrm{SD}$ or as median and range. $\mathrm{A}$ Fisher exact test to compare categoric data was performed. A Pearson correlation coefficient was used for any correlation among the outcome, complications, and age of the patient. A $P$ value $<.05$ was considered statistically significant. Statistics were calculated by using SPSS (Version 21.0; IBM, Armonk, New York). 

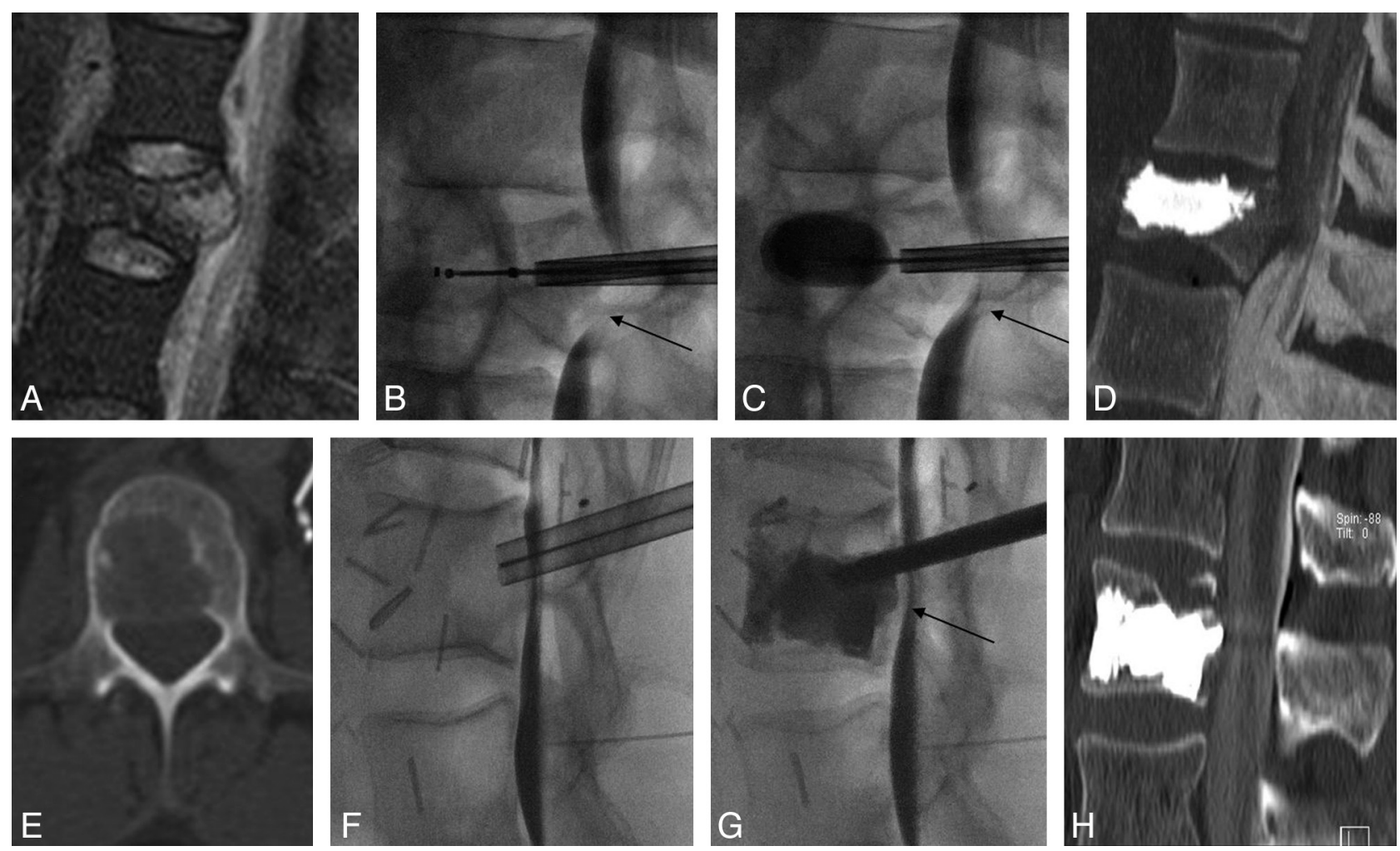

FIG 3. Two cases of the use of intraoperative myelography. $A-D$, Case 1 has a pathologic fracture of $L 1$ related to a renal cell cancer metastatic lytic lesion, characterized by a large posterior wall cortical erosion and a convex epidural mass $(A)$. The intraoperative myelographic images $(B-C)$ show fracture reduction and widening of the central canal during balloon inflation $(C)$, with reduced evidence of myelographic block (compare arrows on $B$ and $C$ ). $D$, Post-cement augmentation reformatted sagittal $C T$ image. $E-H$. Case 2 has a lytic lung cancer metastatic lesion of the $L 2$ vertebral body with a large cortical erosion of the posterior wall $(E)$. Intraoperative myelographic images $(F-G)$ show progressive retropulsion of the posterior aspect of the tumor toward the epidural space, with a posteriorly displaced tumor-contrast interface (arrow on $G$ ) during cement injection in the vertebral body, which prompted halting cement injection. $H$, Final results on CT.

Table 1: Demographic data, imaging characteristics, and vertebral levels treated in 48 patients

\begin{tabular}{lc}
\hline \multicolumn{1}{c}{ Data } & Total \\
\hline Patients (No.) & 48 \\
Age (yr) (mean) ( \pm SD) & $64(14.8)$ \\
Primary cancer (No.) & \\
Solid tumors & 51 \\
Multiple myeloma & 17 \\
Lymphoma & 2 \\
Cortical erosion (No.) & 70 \\
Large & 43 \\
Permeative & 27 \\
Epidural mass (No.) & 31 \\
Large and convex & 18 \\
Small and concave & 13 \\
\hline
\end{tabular}

\section{RESULTS}

Demographic data, tumor histotype, treated levels, and imaging characteristics of the patients of our series are shown in Table 1.

We treated 8 cervical (11.4\%), 38 thoracic (54.3\%), and 24 lumbosacral levels $(34.3 \%)$. The mean number of treated levels per patient was 1.45 (range $=1-5$; median $=1$ ). Thirty patients had 1 level treated, 11 patients had 2 levels treated, 3 patients had 3 levels treated, and 1 patient each had 4 and 5 levels treated during 1 procedure. Compression of neural structures was seen preprocedurally in $16 / 70$ (22.8\%) levels. Of the 45 patients treated, only 18 presented with pain resistant to conservative mea- sures and reasonably attributable to the target lesions that were subsequently treated; in 15 cases, the pain appeared more diffuse and less easily linkable to the target lesions, while in 38 cases stabilization was an exclusive or additional indication to the VA treatment.

No patients in this series had a neurologic deficit attributable to compression of neural structures preprocedurally. An MR imaging-proved EM was present in 31/70 (44.2\%) levels; in 18/ $31(58 \%)$ cases, the EM was large, with a posterior convex profile, while in $13 / 31(41.9 \%)$ cases, it was small, with a posterior concave profile. Combined fluoroscopic/CT guidance was used in $9 / 70(12.8 \%)$ levels, while intraoperative myelography was used in $6 / 70(8.5 \%)$ levels. Balloon kyphoplasty was performed in 7/70 (10\%) levels. Cavity creation with plasma-field-activated radiofrequency (Coblation) wands was used in 59/70 (84.2\%) levels before cement injection. Cement filling of the lytic cavity and adjacent trabecular spaces was desired and attempted, especially in the weight-bearing anterior two-thirds of the vertebral body. According to previously described criteria, ${ }^{9}$ the PMMA filling, as seen on postprocedural CT, was deemed excellent (percentage of filling $=67 \%-100 \%)$ in 40 levels $(57.1 \%)$, good (percentage of filling $=34 \%-66 \%)$ in 25 levels $(35.7 \%)$, and poor (percentage of filling $=0 \%-33 \%)$ in 5 levels $(7.1 \%)$. As a whole, in 65/70 levels (92.8\%), satisfactory PMMA filling (excellent and good) of the lytic cavity and adjacent trabecular spaces was obtained.

An epidural leak of PMMA occurred in 10/70 levels (14.2\%) 

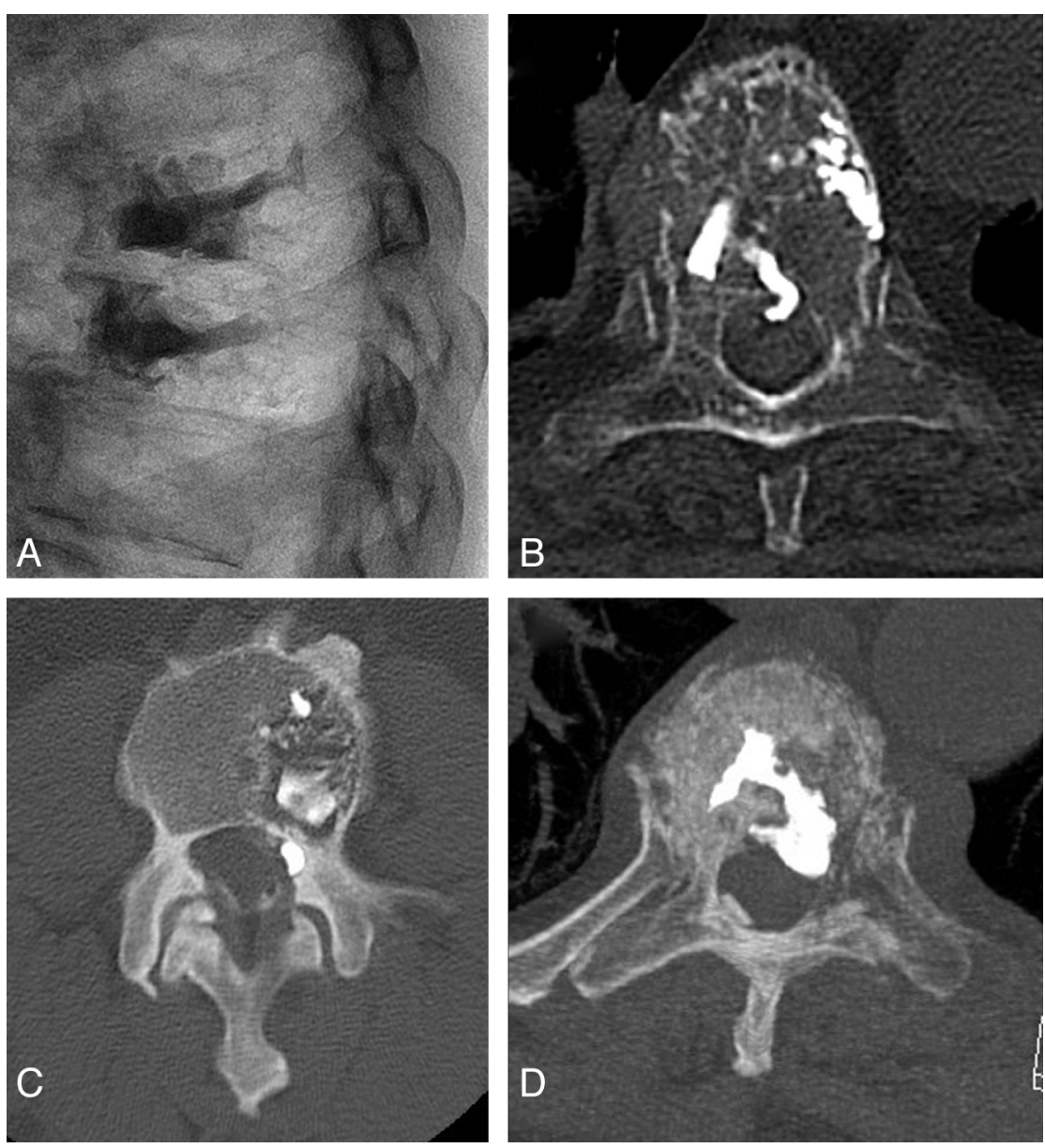

FIG 4. Examples of epidural leaks in our series. $A$ and $B, A$ central ventral epidural leak at T6 in a multiple myeloma vertebral cement augmentation, which was asymptomatic. $C$, A small lateral foraminal epidural leak, causing a transient, self-resolving radicular pain. $D, A$ quite large ventral epidural leak that required stopping the cement injection, resulting in technically unsatisfactory cement filling of the vertebral body. The leak was otherwise clinically silent.

Table 2: Contingency table $(2 \times 2)$ showing the lack of correlation between epidural leak and type of cortical erosion, large or permeative ${ }^{a}$

\begin{tabular}{lccc}
\hline & Epidural Leak+ & Epidural Leak- & Total \\
\hline Large CE & 7 & 36 & 43 \\
Permeative CE & 3 & 24 & 27 \\
Total & 10 & 60 & 70 \\
\hline
\end{tabular}

Note:-CE indicates cortical erosion.

${ }^{a}$ Fisher exact test. Two-tailed $P=.730$.

Table 3: Contingency table $(2 \times 2)$ showing the lack of correlation between epidural leak and presence of epidural mass $^{\mathrm{a}}$

\begin{tabular}{lccc}
\hline & Epidural Leak+ & Epidural Leak- & Total \\
\hline Epidural mass + & 4 & 27 & 31 \\
Epidural mass - & 6 & 33 & 39 \\
Total & 10 & 60 & 70 \\
\hline
\end{tabular}

${ }^{a}$ Fisher exact test. Two-tailed $P=1$.

(Fig 4). In 4/10 leaks (40\%), the patients had an associated EM. No correlation was found between the occurrence of an epidural leak and a permeative or large pattern of cortical erosion (Fisher exact test, $P=.73$ ) (Table 2), between the occurrence of an epidural leak and the presence of an EM (Fisher exact test, $P=1$ ) (Table 3), or between the occurrence of an epidural leak and the type of EM (Fisher exact test, $P=.28$ ) (Table 4). No significant correlation was observed between leak occurrence and tumor histotype ( $\chi^{2}$ test, $\left.P=.805\right)$.

In $3 / 10$ cases with epidural leak, the leak was in the neural foramen; 2 of these patients experienced ipsilateral radicular pain, which spontaneously resolved within 1 week. In 1 case with T1-T2 foraminal leak (Fig 5), the patient developed severe weakness of the right intrinsic hand muscles (myotome T1) without pain; the patient declined decompressive surgery and was treated with oral steroids. The motor deficit partially improved but was nevertheless persistent (M3/5) at 1 month. In the other 7 patients, the epidural leak was in the ventral median and ventral lateral epidural space; no new radicular pain or neurologic deficit occurred postprocedurally or at follow-up.

No patient developed a neurologic deficit related to spinal cord compression. At 1-week and 4-week follow-up, no patients had worsened back or radicular pain attributable to the procedure. In the subgroup of patients with pain judged related to the target level $(n=$ 18), the mean Visual Analog Scale pain score before treatment was $16.3 \pm 2.5$ (range, 12-20) versus $7.4 \pm 4.1$ (range, 1-17) at 1 week versus $6.1 \pm 5.2$ (range, 1-14) at 1 month postprocedurally. One patient who had poor filling at 2 weeks postprocedurally presented with new onset of back pain; imaging revealed increased vertebral collapse at the treated level. This patient underwent a new VA procedure, which was uneventful and successful in obtaining satisfactory PMMA filling and pain resolution (this second procedure was not counted in this series, being a retreatment). In the remaining 4 patients with poor filling reported, no new symptoms were attributable to a clinically significant new or progressive vertebral fracture and their imaging follow-up revealed no increased collapse at 1 month.

\section{DISCUSSION}

In our series of VA procedures in neoplastic lytic vertebral lesions with CE-PW performed for palliation of pain and/or for stabilization, $92.8 \%$ of procedures were technically successful. We observed a cement epidural leak in only $14 \%$ of levels, despite the presence of the CE-PW in all patients of the cohort by definition of the inclusion criteria. The rate of transient clinical complications was extremely low (4.2\%), but 1 (1.4\%) clinical complication with permanent disability, likely from radicular injury related to a PMMA foraminal leak, did occur. Notably, none of the patients developed a neurologic deficit related to spinal cord com- 
pression. We also found that the presence of an MR imagingproved EM did not represent a risk factor for epidural leak.

\section{Treatment Indications}

The aim of the VA procedure was pain palliation and/or stabilization in fractured or at-risk-of-collapse vertebral bodies. The indication to treat with VA was presented for each individual patient in the setting of a multidisciplinary discussion (Spine Tumor Board), where conservative, medical, radiation, surgical, and interventional radiology therapeutic options were discussed. Whenever stability was a concern but surgery was contraindicated by the general condition of the patient or by the indication not to delay radiation treatment or by local specific conditions (ie, multilevel metastatic involvement), either reasonable stabilization was deemed obtainable by a less invasive percutaneous cement augmentation procedure or VA was favored as a first-line thera-

Table 4: Contingency table $(2 \times 2)$ showing the lack of correlation between of epidural leak and type of epidural mass, "large convex" or "small concave"a

\begin{tabular}{lccc}
\hline & Epidural Leak+ & Epidural Leak- & Total \\
\hline Epidural mass S CV & 3 & 10 & 13 \\
Epidural mass L CX & 1 & 17 & 18 \\
Total & 4 & 27 & 31 \\
\hline
\end{tabular}

Note:- S CV indicates small concave; L CX, large convex.

${ }^{a}$ Fisher exact test. Two-tailed $P=.283$.
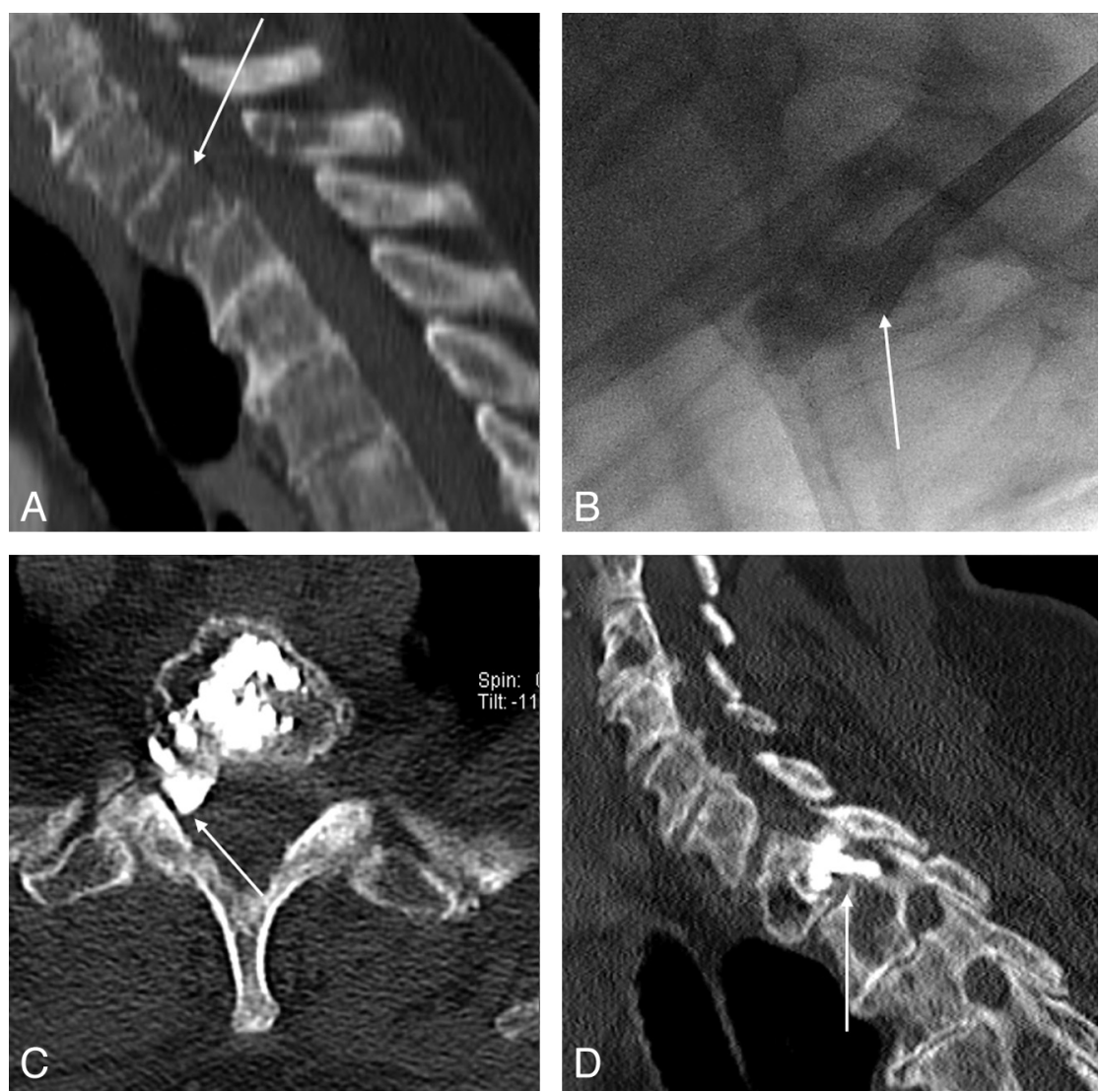

FIG 5. Symptomatic T1-T2 foraminal cement leak. Extensive breast cancer metastatic lytic lesion of the Tl vertebral body with a large cortical erosion of the posterior wall (arrow on A). Vertebral augmentation was performed under fluoroscopic guidance (B); despite inherently poor visibility in the lateral view of the cervicothoracic junction, the epidural leak was promptly recognized (arrow on B) and cement injection was halted. Nevertheless, the strategically located right foraminal PMMA leak (arrows on $C$ and $D$ ) caused a permanent motor deficit of the 71 myotome. peutic approach. In patients with multilevel involvement, selection of levels to treat to prevent a fracture was based on the extent and location of the lytic lesions suggesting biomechanical risk of collapse as described by Krishnaney et $\mathrm{al}^{20}$ and Taneichi et $\mathrm{al}^{21}$ and by the Spinal Instability Neoplastic Score criteria. ${ }^{11}$ In patients with pain and multilevel vertebral neoplastic involvement, identifying the lesion or the cause of the symptoms is often not possible with certainty. In such cases, the selection of levels to treat with VA was based on clinical judgment, in some cases aided by physical examination under fluoroscopy.

Worldwide, radiation therapy is still the criterion standard for spinal metastases. Nevertheless, there are non-radiation-sensitive tumors, $>20 \%-30 \%$ of patients with painful bone metastasis are nonresponders, and radiation therapy may not be an option because of high radiation doses previously delivered. Moreover, radiation treatment requires a 2 - to 4 -week delay to achieve maximum effect; and following radiation, there might be a phase of increased vertebral fragility and risk of fracture. ${ }^{22}$ VA can be included in the multimodal treatment of spinal metastases with a role complementary to radiation therapy, offering prompt pain relief and immediate stabilization of the anterior column. In selected cases, if VA is successful in relieving pain and/or offering stability and if there is no real curative goal, radiation therapy can be avoided or the dose exposure spared for a later time. Hirsch et $\mathrm{al}^{23}$ reported no difference in pain outcomes with regard to sequencing of radiation therapy and the VA procedure. In addition, clinical use of radiation against neoplastic disease is not affected by the presence of PMMA, and the characteristics of PMMA are not affected by the level of the radiation dose in clinical use. At our institution, the presence of neurologic deficits due to compression of nerve structures does not absolutely preclude VA, but radiation treatment or decompressive surgery or both are rather considered as first-line treatments and VA is sometimes performed in conjunction or as a second-line stabilization or pain-palliation measure. In this series, none of the treated patients presented with neurologic deficits due to spinal cord compression before the procedure.

\section{Procedure Efficacy}

The aim of the VA procedure was to fill the lytic areas in the weight-bearing portions of the vertebral body with PMMA, namely the anterior two-thirds, also to obtain cement interdigitation in the nonlytic adjacent trabecular bone, whenever possible.

Because some patients included in this study did not present with pain and underwent the VA procedure exclu- 
sively for stabilization, we did not assess pain levels as a measure of efficacy of the procedure. Nevertheless, in the subgroup of patients presenting with pain and a correlated target lesion, VA resulted in significant pain reduction at 1 week and 1 month, reproducing known results from the literature. ${ }^{10-13}$ Because the study is retrospective and the patients were not specifically selected for inclusion on the basis of pain symptoms only and because individual patients received different pain pharmacologic regimens and different oncologic treatment regimens, the reported results on pain might reflect confusing effects from other factors not assessed in this study. As a measure of the efficacy of the procedure, we focused rather on the degree of PMMA filling and the rate of technical and clinical complications related to the procedure. Cement leakage into the spinal canal or neuroforamina (Figs 4 and 5), one of the most feared technical complications of VA in neoplastic vertebral lesions due to its potential for nerve damage, was assessed by technical and clinical complications of the procedure. We did not perform imaging follow-up of those levels in which we achieved good or excellent cement filling. The efficacy of the stabilization procedure in arresting or preventing clinically significant vertebral collapse was rather inferred by the absence of new clinical symptoms at the target levels. In patients with poor filling who underwent the 1-month plain film followup, no new collapse was detected. Only 1 patient among those with poor PMMA filling presented within 1 month postprocedurally with new back pain, prompting imaging assessment that disclosed increased collapse at the treated level.

\section{VA Technique}

Due to the highly variable characteristics of the vertebral lesions requiring treatment, each posing specific technical challenges, the VA procedures in this series have been performed by using different techniques, imaging-guidance modalities, tools, and devices, individualized for each case.

Fluoroscopy was the imaging-guidance technique of choice, invariably used to have real-time control of PMMA injection, but in cases with extensive lytic disruption of the pedicles or poor fluoroscopic visibility of bony landmarks, CT guidance was used to place the needles and to intermittently control PMMA spread. When posterior wall retropulsion or a large EM was encroaching on the spinal canal, intraoperative myelography allowed indirect visualization of the posterior vertebral body margins and depicted intraprocedural worsening of retropulsion. This technique was applicable only at the lumbar and lower thoracic levels, where the contrast agent injected intrathecally would form a stable and visible contrast-tumor interface in the prone patient. Intraoperative myelography increased operator comfort and confidence in such challenging cases, and in only 1 case did it actually show a progressive mild retropulsion of soft-tissue tumor during cement injection (Fig $3 E-H$ ). This observation led to halting the cement injection; the cement filling was already satisfactory and no clinical consequences ensued. In no other case was an evident softtissue-mass posterior displacement noted as an immediate consequence of the procedure.

Every effort was made to allow the most optimal and precise fluoroscopic projections and visualization throughout each phase of the VA procedure; we think this effort is crucial in such challenging cases.

All the VA procedures used high-viscosity PMMA, with a working time of approximately 18 minutes. PMMA injection was performed very slowly, starting as anterior as possible in the vertebral body, with mandatory real-time continuous fluoroscopic control in the lateral projection and intermittent anteroposterior checks. The use of high-viscosity cement reduces the rate of extravertebral leaks. ${ }^{17}$ The volumes of injected PMMA varied widely; this variation reflected differences in the size of lytic lesions, trabecular compliance, and injected cement distribution. ${ }^{24}$

\section{Cavity Creation}

In most cases in our series, due to the presence of CE-PW, cavity creation in the anterior two-thirds of the vertebral body was performed before cement injection, to favor optimal PMMA filling of the lytic cavity while minimizing the risks of epidural PMMA leak or displacement of solid tumoral tissue through the dehiscent posterior wall. ${ }^{25}$ Cavity creation in the vertebral body was performed with Coblation Wands (Fig 2), as previously reported by other authors, ${ }^{19,26,27}$ in 59/70 levels. The decision not to use Coblation was made intraprocedurally when the advancement of the trocar cannulas through the lytic area did not reveal typical softtissue consistency, but rather fluid consistency, such as in markedly necrotic tumors or in some multiple myeloma lesions.

Coblation is a form of radiofrequency energy that, through a controlled, non-heat-driven process, excites the electrolytes in a conductive medium, such as saline solution. ${ }^{28}$ The energized particles cause tissue to dissolve into gas at relatively low temperatures with volumetric removal of target tissue with minimal damage and minimal heating of the surrounding tissue. ${ }^{29}$ Dissolution through vaporization of soft tissues in close proximity of the tip of the Coblation Wand, with a modest temperature rise, represents a theoretic advantage over conventional thermal radiofrequency tumor ablation, also used in combination with VA in metastatic lesions of the spine. ${ }^{26}$ Radiofrequency kills the tumor tissue without dissolving it, therefore without truly creating a cavity; moreover, radiofrequency causes a significant rise in the temperature of the soft tissues and renders neural structures at risk of heat lesions, especially when the posterior wall cortex is defective. ${ }^{26}$ The creation of a cavity by tissue dissolution also represents a theoretic advantage over a cavity created by mechanical tissue displacement, as in kyphoplasty. We used kyphoplasty in 7 cases, not for cavity creation but always after the cavity was created with Coblation, in an attempt to obtain height restoration and reduction of posterior wall retropulsion (Fig $3 A-D$ ), exploiting the tension of the posterior longitudinal ligament (ligamentotaxis). ${ }^{30}$

\section{Complications}

CE-PW is thought to be a risk factor for epidural PMMA leak, which has potential for nerve structure compression and neurologic injury and might require decompression surgery. Furthermore, a leak occurring early during injection would prompt the early interruption of the procedure, limiting the desired filling of the vertebral body. Despite the potential for nerve damage, in most cases, the leakage is asymptomatic or manifests as transient 
radicular pain, ${ }^{9}$ which is due to PMMA-related heating rather than compressive damage.

In the only patient in our series who reported a permanent clinical complication related to the procedure, a foraminal PMMA leak occurred at T1-T2 (Fig 5). It was a breast cancer metastasis involving the T1 vertebra. Coblation-assisted cavity creation was performed. Despite the inherent relatively poor fluoroscopic visibility of the cervicothoracic junction on lateral-view fluoroscopy during the PMMA injection, the posterior cement leak was promptly recognized and cement injection was stopped. The patient presented a few hours later with a radicular T1 motor deficit. No spinal cord compression was noted. Conservative-versus-decompressive surgery measures were discussed, and the patient elected not to undergo surgery.

The occurrence of cement leakage in our series (14\%) only in patients with CE-PW was not larger than that in other published series on neoplastic lytic lesions, ${ }^{9,13,17}$ and it is well-recognized in the literature that the depiction of PMMA leaks is much higher when CT is used as a postprocedural imaging follow-up compared with fluoroscopy or plain films. Similarly, the notably low rate of clinical complication (3 cases, [4.2\%], 2 of which were transient) obtained in our study is the result of multiple factors. We believe that creation of a well-sized cavity by using Coblation allows low-pressure injection, makes the distribution of PMMA more predictable, and can effectively limit the chance of posterior cement leakage in this subcategory of patients. Use of high-viscosity PMMA, optimal fluoroscopic visualization of the field, and appropriate knowledge of radiographic and cross-sectional spinal anatomy are key factors. To validate the results of this singlecenter single-operator study, a larger, multicenter study with a larger cohort and longer follow-up may be useful.

A limitation of our study is the retrospective nature, even though this cohort is the result of a prospectively established data base and all the cases performed were included. Lack of a control group and lack of long-term imaging or clinical follow-up to assess durability in all patients were other limitations.

\section{CONCLUSIONS}

Our data seem to justify the use of percutaneous vertebral augmentation in neoplastic lytic vertebral lesions with cortical erosion of the posterior wall, performed for palliation of pain and/or for stabilization in case of risk of vertebral collapse. Adequate skills levels; state-of-the-art equipment, devices, and materials; and individualized technical adjustments are critical for keeping complication rates low.

Disclosures: Michal Reinert-UNRELATED: Grants/Grants Pending: Oxygen Biotherapeutics, ${ }^{\star}$ Comments: severe head injury studies. *Money paid to the institution.

\section{REFERENCES}

1. Jacobs WB, Perrin RG. Evaluation and treatment of spinal metastases: an overview. Neurosurg 2001;11:e10

2. Jemal A, Thun MJ, Ries LA, et al. Annual report to the nation on the status of cancer, 1975-2005, featuring trends in lung cancer, tobacco use, and tobacco control. J Natl Cancer Inst 2008;100: 1672-94

3. Kassamali RH, Ganeshan A, Hoey ET, et al. Pain management in spinal metastases: the role of percutaneous vertebral augmentation. Ann Oncol 2011;22:782-86
4. Galibert P, Deramond H, Rosat P, et al. Preliminary note on the treatment of vertebral angioma by percutaneous acrylic vertebroplasty [in French]. Neurochirurgie 1987;33:166-68

5. Chiras J, Barragán-Campos HM, Cormier E, et al. Vertebroplasty: state of the art [in French]. J Radiol 2007;88:1255-60

6. Tancioni F, Lorenzetti MA, Navarria P, et al. Percutaneous vertebral augmentation in metastatic disease: state of the art. J Support Oncol 2011;9:4-10

7. Weill A, Chiras J, Simon JM, et al. Spinal metastases: indications for and results of percutaneous injection of acrylic surgical cement. Radiology 1996;199:241-47

8. Deramond H, Depriester C, Galibert P, et al. Percutaneous vertebroplasty with polymethylmethacrylate: technique, indications, and results. Radiol Clin North Am 1998;36:533-46

9. Barragán-Campos HM, Vallee JN, Lo D, et al. Percutaneous vertebroplasty for spinal metastases: complications. Radiology 2006; 238:354-62

10. Dudeney S, Lieberman IH, Reinhardt MK, et al. Kyphoplasty in the treatment of osteolytic vertebral compression fractures as a result of multiple myeloma. J Clin Oncol 2002;20:2382-87

11. Fourney DR, Schomer DF, Nader R, et al. Percutaneous vertebroplasty and kyphoplasty for painful vertebral body fractures in cancer patients. J Neurosurg 2003;98:21-30

12. Berenson J, Pflugmacher R, Jarzem P, et al. Balloon kyphoplasty versus non-surgical fracture management for treatment of painful vertebral body compression fractures in patients with cancer: a multicentre, randomised controlled trial. Lancet Oncol 2011;12: 225-35

13. van der Linden E, Kroft LJ, Dijkstra PD. Treatment of vertebral tumor with posterior wall defect using image-guided radiofrequency ablation combined with vertebroplasty: preliminary results in $\mathbf{1 2}$ patients. J Vasc Interv Radiol 2007;18:741-47

14. Trial C, Brancati A, Marnet O, et al. Coblation technology for surgical wound debridement: principle, experimental data, and technical data. Int J Low Extrem Wounds 2012;11:286-92

15. Gerszten PC, Monaco EA 3rd. Complete percutaneous treatment of vertebral body tumors causing spinal canal compromise using a transpedicular cavitation, cement augmentation, and radiosurgical technique. Neurosurg Focus 2009;27:E9

16. Wilson DJ, Owen S, Corkill RA. Coblation vertebroplasty for complex vertebral insufficiency fractures. Eur Radiol 2013;23:1785-90

17. Anselmetti GC, Zoarski G, Manca A, et al. Percutaneous vertebroplasty and bone cement leakage: clinical experience with a new high-viscosity bone cement and delivery system for vertebral augmentation in benign and malignant compression fractures. Cardiovasc Intervent Radiol 2008;31:937-47

18. Boulter DJ, Rumboldt Z, Bonaldi G, et al. Tilting the gantry for CTguided spine procedures. Radiol Med 2014;119:750-57

19. Georgy BA, Wong W. Plasma-mediated radiofrequency ablation assisted percutaneous cement injection for treating advanced malignant vertebral compression fractures. AJNR Am J Neuroradiol 2007;28:700-05

20. Krishnaney AA, Steinmetz MP, Benzel EC. Biomechanics of metastatic spine cancer. Neurosurg Clin North Am 2004;15:375-80

21. Taneichi $\mathrm{H}$, Kaneda K, Takeda N, et al. Risk factors and probability of vertebral body collapse in metastases of the thoracic and lumbar spine. Spine 1997;22:239-45

22. Rose PS, Laufer I, Boland PJ, et al. Risk of fracture after single fraction image-guided intensity-modulated radiation therapy to spinal metastases. J Clin Oncol 2009;27:5075-79

23. Hirsch AE, Jha RM, Yoo AJ, et al. The use of vertebral augmentation and external beam radiation therapy in the multimodal management of malignant vertebral compression fractures. Pain Physician 2011;14:447-58

24. Tschirhart CE, Finkelstein JA, Whyne CM. Optimization of tumor volume reduction and cement augmentation in percutaneous vertebroplasty for prophylactic treatment of spinal metastases. J Spinal Disord Tech 2006;19:584-90 
25. Ahn H, Mousavi P, Chin L, et al. The effect of pre-vertebroplasty tumor ablation using laser-induced thermotherapy on biomechanical stability and cement fill in the metastatic spine. Eur Spine $J$ 2007;16:1171-78

26. Georgy BA. Bone cement deposition patterns with plasma-mediated radio-frequency ablation and cement augmentation for advanced metastatic spine lesions. AJNR Am J Neuroradiol 2009;30: 1197-202

27. Groetz SF, Birnbaum K, Meyer C, et al. Thermometry during coblation and radiofrequency ablation of vertebral metastases: a cadaver study. Eur Spine J 2013;22:1389-93
28. Bortnick DP, Plastic Surgery Educational Foundation DATA Committee. Coblation: an emerging technology and new technique for soft-tissue surgery. Plast Reconstr Surg 2001;107: $614-15$

29. Chen YC, Lee SH, Saenz Y, et al. Histologic findings of disc, end plate and neural elements after coblation of nucleus pulposus: an experimental nucleoplasty study. Spine J 2003;3:466-70

30. Bonaldi G, Cianfoni A. Percutaneous treatment of lumbar compression fracture with canal stenosis and neurogenic intermittent claudication: combining kyphoplasty and interspinous spacer. $J$ Vasc Interv Radiol 2012;23:1437-41 